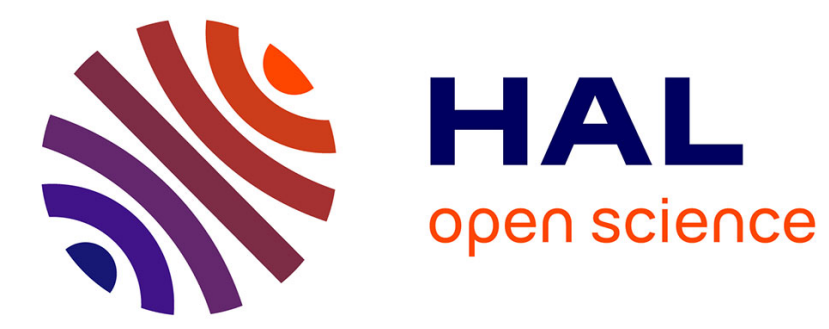

\title{
Laboratory estimation of apparent soil thermal conductivity using a numerical approach
}

\author{
Laurent Bruckler, P. Renault, Franck Ariès
}

\section{To cite this version:}

Laurent Bruckler, P. Renault, Franck Ariès. Laboratory estimation of apparent soil thermal conductivity using a numerical approach. Soil Science, 1987, 143 (6), pp.387-397. hal-02721345

\section{HAL Id: hal-02721345 \\ https://hal.inrae.fr/hal-02721345}

Submitted on 1 Jun 2020

HAL is a multi-disciplinary open access archive for the deposit and dissemination of scientific research documents, whether they are published or not. The documents may come from teaching and research institutions in France or abroad, or from public or private research centers.
L'archive ouverte pluridisciplinaire HAL, est destinée au dépôt et à la diffusion de documents scientifiques de niveau recherche, publiés ou non, émanant des établissements d'enseignement et de recherche français ou étrangers, des laboratoires publics ou privés. 


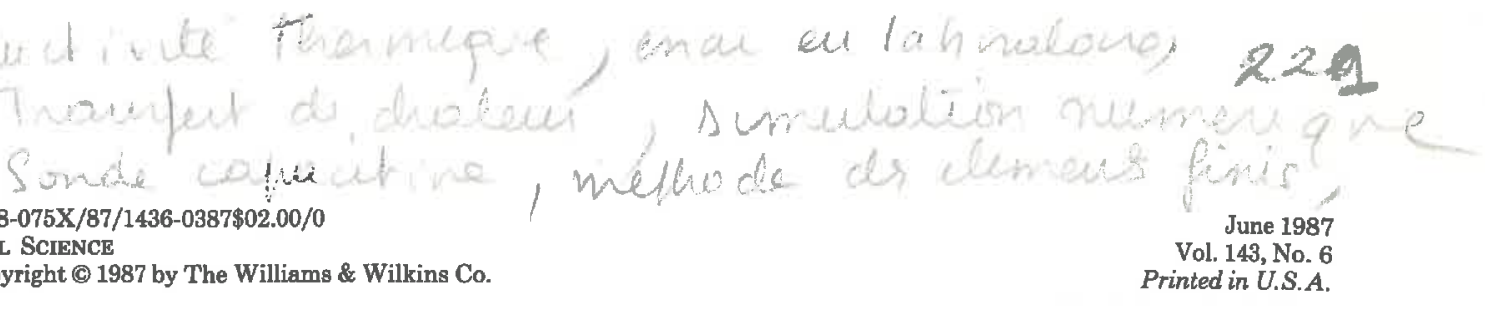

\section{LABORATORY ESTIMATION OF APPARENT SOIL THERMAL CONDUCTIVITY USING A NUMERICAL APPROACH}

\section{BRUCKLER, ${ }^{1}$ P. RENAULT, ${ }^{1}$ AND F. ARIES ${ }^{2}$}

The experimental equipment used in the laboratory is a metal cylinder with its top and its base, containing a soil sample. A sensitive temperature probe is placed at the center of the soil, and temperture data are recorded versus time during a transient heat flow. The estimate of the thermal conductivity is provided according to a numerical approach for solving the heat conduction equation, using the finite element method. This numerical approach takes into account the heat transfer in all the components of the apparatus (metal wall, porous medium, temperature probe, and its wire) and in all directions (axial and radial fluxes). Comparing calculated values and experimental data gives a criterion that shows how the modeling fits. We first present numerical and experimental results from isotropic soil samples. The chosen modeling fits very well, and we regard estimated values of thermal conductivity as satisfactory. Secondly we present a theoretical and numerical approach to calculating two thermal conductivities for an anisotropic soil (thermal conductivities in the radial and axial directions). For this purpose, we combine the finite element method with a nonlinear fitting method and show that two temperature probes are needed in the soil sample, and that their locations are optimized according to numerical simulations.

There are numerous laboratory methods of measuring apparent thermal conductivity. Generally different authors agree upon the following points: (1) in measurements concerning the apparent thermal conductivity, the term apparent is due to the fact that the heat conducted by the water vapor is not separated from the total heat flux; (2) the transient state flow is regarded as

${ }^{1}$ INRA, Station de Science du Sol, B.P. 91, Domaine St Paul, 84140 Montfavet, France.

${ }^{2}$ INRA, Station de Biometrie, B.P. 91, Domaine St Paul, 84140 Montfavet, France.

Received for publication 23 Jan. 1986; revised 9 June 1986. the best method of measurement, because the steady-state heat conduction corresponds generally to a heterogeneous medium in terms of water content distribution in the porous media, and that occurs because the coupled heat and mass transfers induce both water content and temperature gradients that become important after long durations.

The classical methods of measuring have some advantages and disadvantages

1. Where the authors have an analytical solution for the thermal conductivity value (Barnes et al. 1981; Moench and Evans 1970; Goldsmid et al. 1981; Rama Mohan and Raghavendra 1973), calculations are very quick. But they don't always use a criterion of comparison between the predicted temperatures or fluxes and the experimental temperatures or fluxes in the apparatus. Thus, it is impossible to know if the solution is adequate or not.

2. The hypotheses made are sometimes very strong, e.g., the medium is considered homogeneous and isotropic. It is well known that this hypothesis is sometimes false, especially when the soil sample in the apparatus has structural layers. Also, where the equipment is a cylinder, axial heat conduction is regarded as negligible. Thus, the authors try to adapt the equipment to the analytical solution (a cylinder small in diameter), but it would be better to adapt the mathematical procedure to fit the most convenient experimental apparatus. Experimental methods sometimes need the measurements of temperatures and heat fluxes. Methods that need measurements of only temperatures or heat fluxes seem to be better because the sources of measurement errors are limited.

In this paper, we present a numerical procedure to calculate apparent thermal conductivity using the finite element method. This method is more time consuming than the analytical solution, but it presents some important advantages - It makes it possible to take into account 
axial and radial heat conduction in the cylinder, and to take into account the thermal conductivity of both the walls of the cylinder and of the wire of the temperature probe at the center of the cylinder.

-It makes it possible to develop a mathematical procedure when the medium is regarded as anisotropic.

-The comparison between calculated and experimental values produced allows us to know if the model is adequate or not.

\section{THEORY}

For an anisotropic soil with principal directions $r$ and $y$, Fourier's law can be written as

$$
q_{r}=-\lambda_{r} \frac{\partial T}{\partial r}
$$

and

$$
q_{y}=-\lambda_{y} \frac{\partial T}{\partial y}
$$

where

$q_{r}, q_{y}=$ heat fluxes in the $O_{r}$ and $O_{y}$ directions, $\mathrm{W} \mathrm{m}^{-2}$;

$\lambda_{r}, \lambda_{y}=$ thermal conductivities in the $O_{r}$ and $O_{y}$ directions, $\mathrm{W} \mathrm{m}^{-1} K^{-1}$; and

$\frac{\partial T}{\partial r}, \frac{\partial T}{\partial y}=$ temperature gradients in the $O_{r}$ $\frac{\partial T}{\partial r}, \frac{\partial T}{\partial y}=$ and $O_{y}$ directions, $\mathrm{K} \cdot \mathrm{m}^{-1}$.

For an axially symmetric heat flow, the heat balance can be written as

$$
\rho C_{p} \frac{\partial T}{\partial t}=-\frac{1}{r} \frac{\partial}{\partial r} r q_{r}-\frac{\partial}{\partial y} q_{y}
$$

where $\rho=$ specific mass of the material, $\mathrm{kg} \cdot \mathrm{m}^{-3}$, and $C_{p}=$ specific heat capacity, $\mathrm{J} \cdot \mathrm{kg}^{-1} \cdot \mathrm{C}^{-1}$.

Combining Eqs. (1), (2), and (3) gives

$$
\begin{aligned}
& \rho C_{p} \frac{\partial T}{\partial t}=\frac{1}{r} \\
& \cdot \frac{\partial}{\partial r}\left[r \cdot \lambda_{r} \cdot \frac{\partial T}{\partial r}\right]+\frac{\partial}{\partial y}\left[\lambda_{y} \cdot \frac{\partial T}{\partial y}\right]
\end{aligned}
$$

Multiplying the two sides of Eq. (4) by $r$, and given that

$$
\begin{aligned}
& C_{p} \cdot r=C_{p}{ }^{r} \\
& \lambda_{r} \cdot r=\lambda_{r}{ }^{r} \\
& \lambda_{y} \cdot r=\lambda_{y}{ }^{r}
\end{aligned}
$$

Eq. (4) becomes

$$
{ }_{\rho} C_{p}{ }^{r} \frac{\partial T}{\partial t}=\frac{\partial}{\partial r}\left[\lambda_{r}{ }^{r} \frac{\partial T}{\partial r}\right]+\frac{\partial}{\partial y}\left[\lambda_{y}{ }^{r} \frac{\partial T}{\partial y}\right]
$$

For an isotropic soil $\lambda_{r}=\lambda_{y}=\lambda$; thus $\lambda_{r}{ }^{r}=$ $\lambda_{y}{ }^{r}=\lambda^{r}$, and Eq. (6) reduces to the simple and symmetric form

$$
\rho C_{p}{ }^{r} \frac{\partial T}{\partial t}=\frac{\partial}{\partial r}\left[\lambda^{r} \frac{\partial T}{\partial r}\right]+\frac{\partial}{\partial y}\left[\lambda^{r} \frac{\partial T}{\partial y}\right]
$$

Equation (6) or Eq. (7), to be solved, requires only heat conduction in the soil materials. In fact, it is well known that temperature gradients and moisture gradients invariably exist together, and the satisfactory mathematical solution is then to solve heat and mass transfers in porous media. However, in this last case too many unknown parameters must be taken into account to hope to obtain a good and simple estimation of the thermal conductivity of soils.

\section{MATERIALS AND METHODS}

Soil samples are encased in a metal cylinder (14.0 cm long, $8.5 \mathrm{~cm}$ in outside diameter, 6.8 $\mathrm{cm}$ in internal diameter) that contains at its center a sensitive temperature probe $(0.3 \times 0.8$ $\mathrm{cm})$. The complete equipment requires two water baths (Fig. 1). The first one, which has a precision of $0.1^{\circ} \mathrm{C}$, is used for a preliminary thermal equilibrium (temperature $T_{1}, 25^{\circ} \mathrm{C}$ ) of

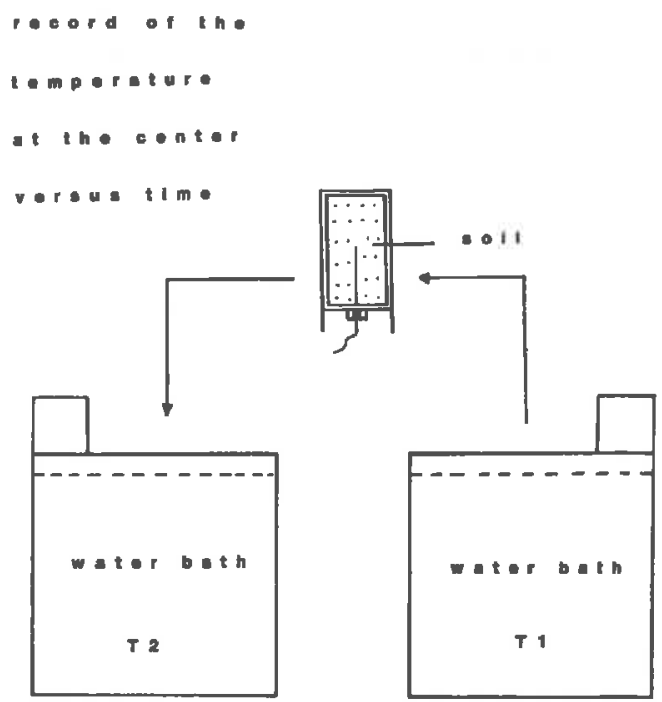

Fig. 1. Equipment used for measuring the apparent thermal conductivity. 
the metal cylinder with the soil sample. At time 0 , the cylinder is placed in the second water bath (temperature $T_{2}, 20^{\circ} \mathrm{C}$ ), which has a precision of $0.05^{\circ} \mathrm{C}$ and gives a constant temperature all around the metal cylinder. The measurements of the temperature at the center of the cylinder versus time are recorded with automatic equipment. The calibration of the temperature probe has been made between $15-35^{\circ} \mathrm{C}$ using highprecision thermometers. Figure 2 shows the residuals of the temperature obtained with a parabolic curve fitting. All the residuals are in the interval $-0.04-+0.04^{\circ} \mathrm{C}$. Thus, we consider that the maximum absolute error for temperature measurements due to the use of the fitted curve is less than $0.04^{\circ} \mathrm{C}$.

Soil samples are generally composed of aggregates $(2-3 \mathrm{~mm})$ at a given volumetric water content. The samples are prepared by adding the amount of water required to bring a dry sample to a desired water content. Required amounts of water were sprinkled into the soil, which was then shaken in a plastic bag and kept in a room for several days to allow uniform distribution of water. At the end of the experiment, determinations of the bulk density and water content were made. The heat capacity is estimated using a classical approach in which the total heat capacity of the medium requires knowledge of the fractional volumetric components of solid, water, and organic matter. For

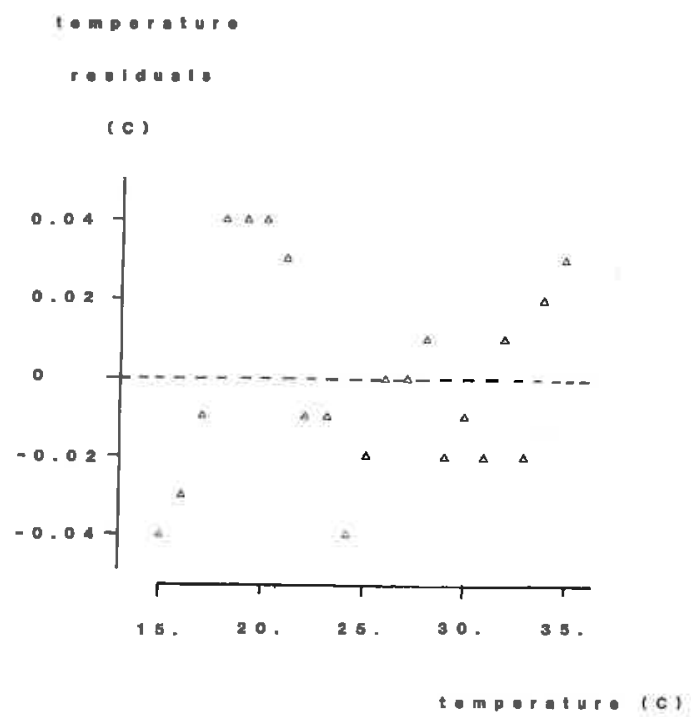

FIG. 2. Temperature residuals of the probe after calibration. determinations of thermal conductivity under compacted conditions, a piston allows the soil in the cylinder to be compacted to the desired degree.

\section{MATHEMATICAL PROCEDURE AND CalCULations}

A numerical solution for the heat conduction equation has been obtained by using the finite element method for solving a second-order partial differential equation applied to either an isotropic or anisotropic porous medium.

In this paper, we consider the thermal properties of the experimental equipment as consisting of three component mediums

1. the metal cylinder (Medium 1), including its top and base

2. the soil sample (Medium 2), which may be either isotropic, $\left(\lambda_{r}=\lambda_{y}\right)$, or anisotropic, $\left(\lambda_{r} \neq \lambda_{y}\right)$

3. the temperature probe (Medium 3), including its wire, running from the center of the soil to the cylinder base.

The calculations are then made by solving Eq. (6) for these three media under either isotropic or anisotropic conditions of the soil (Medium 2).

The half-grid layout used for the numerical computation is shown in Fig. 3. It is composed of 512 finite elements shaped like triangles; those lying nearest the boundaries of the component mediums are smaller. The total number of nodes is 292 , but 47 of them represent the external metal wall and have a constant temperature considered as instantaneously equal to the temperature of the second water bath. This leaves 245 unknown values to be solved at each given time step by a linear equation system. After numerical computations, the calculated temperatures at the center of the cylinder for various iterative thermal conductivity values and the temperature data produced experimentally are compared. This comparison is used as a criterion to verify the adequacy of the numerical modeling.

\section{Preliminary calibration of the numerical modeling}

Modeling of heat conduction applied to an ensemble of the three mediums, each having different physical properties, requires those properties - density, heat capacity, and thermal conductivity-to be known for each medium. 


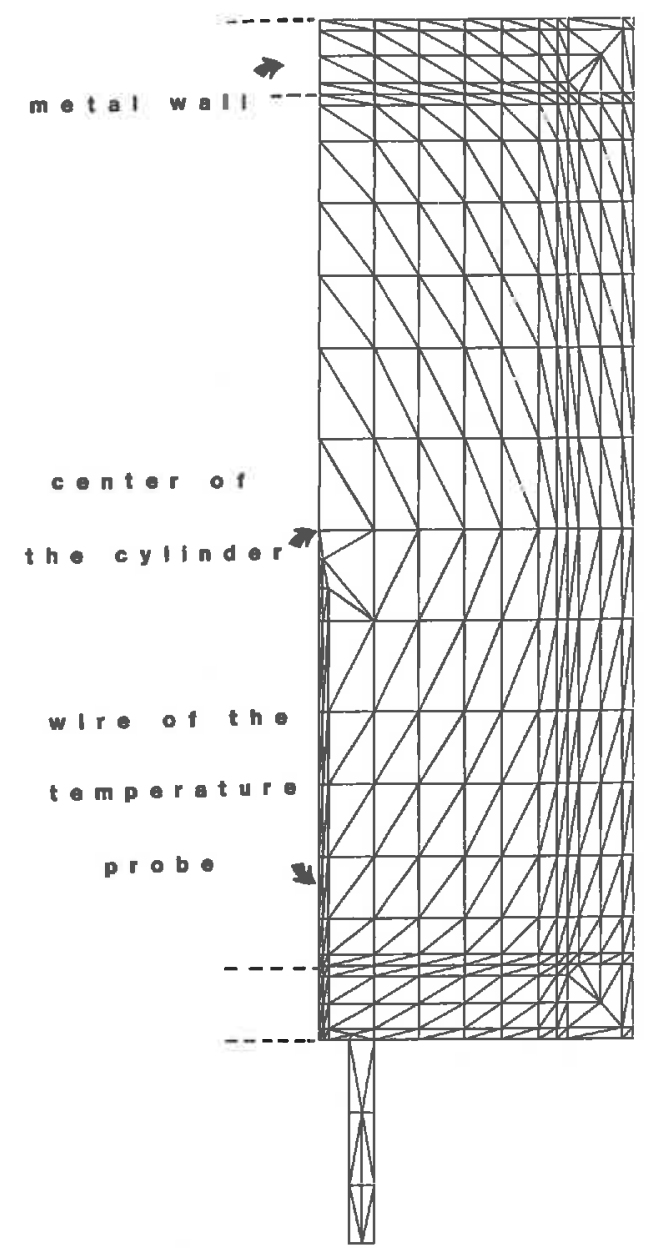

FIG. 3. The half-grid layout using finite elements.
The thermal conductivity of the soil medium is the objective, but the other parameters must first be determined. The physical properties for the soil are measured for each experiment, but the physical properties of the cylinder wall and soil probe are regarded as constant values, once having been determined. Based on four measurements, the interval of confidence for the experimentally derived values of the density and heat capacity of the metal cylinder walls are $8.89-8.94 \mathrm{~g} / \mathrm{cm}^{3}$ and $0.092-0.093 \mathrm{cal} \mathrm{g}^{-1} \mathrm{C}^{-1}$, respectively. These support values are very close to the values for copper $\left(8.91 \mathrm{~g} / \mathrm{cm}^{3}\right.$ and 0.092 cal $\mathrm{g}^{-1} \mathrm{C}^{-1}$ ), the metal cylinder having a $93 \%$ copper content. The thermal conductivity value of the cylinder is thereby chosen as that of copper, a decision based also upon the very low sensitivity of the modeling to this value. Values for the physical properties of the temperature probe are, however, relatively difficult to measure. For this reason, the density and heat capacity values of the temperature probe are chosen to be the same as those for the metal cylinder wall, and the thermal conductivity of the probe is estimated by minimizing the differences between results from calculated values and results from experimental data (i.e, residuals) for a dry and noncompacted soil in the cylinder. Thus, using this calibration procedure, we estimate the two thermal conductivities for the dry, noncompacted soil sample and the probe simultaneously by minimizing the differences between calculated and experimental values. As shown in Table 1 , the mean residual value obtained after

TABLE 1

Calibration of the wire thermal conductivity: values of the mean residuals of the temperature at the center of the cylinder $\left({ }^{\circ} \mathrm{C}\right)$

\begin{tabular}{cccccc}
\hline $\begin{array}{c}\text { Wire thermal } \\
\text { conductivity, } \\
\mathbf{W ~ m}^{-1} \mathbf{K}^{-1}\end{array}$ & \multicolumn{5}{c}{$\begin{array}{c}\text { Dry soil thermal conductivity, } \\
\mathbf{W ~ m}^{-1} \mathrm{~K}^{-1}\end{array}$} \\
\cline { 2 - 5 } 90 & 0.132 & 0.133 & 0.134 & 0.135 & 0.136 \\
100 & & & 0.056 & 0.042 & 0.039 \\
110 & & & 0.048 & 0.036 & 0.037 \\
120 & & 0.055 & 0.040 & 0.034 & 0.040 \\
130 & & 0.047 & 0.035 & 0.035 & 0.046 \\
140 & 0.040 & 0.033 & 0.039 & 0.056 \\
150 & 0.048 & 0.035 & 0.036 & 0.047 & 0.063 \\
160 & 0.041 & 0.034 & 0.040 & 0.053 & \\
170 & 0.038 & 0.037 & & & \\
180 & 0.037 & 0.039 & & & \\
190 & 0.037 & & & & \\
200 & 0.039 & & & & \\
\hline
\end{tabular}


calibration is about $0.03^{\circ} \mathrm{C}$, which is very small. Furthermore, the estimated value for the thermal conductivity of the wire $\left(140 \mathrm{~W} \mathrm{~m}^{-1} \mathrm{~K}^{-1}\right)$ seems physically reasonable, because this probe is a plastic-insulated copper wire, and, thus, the corresponding estimated value must be less than the thermal conductivity of the pure metal. According to the relatively low sensitivity of the modeling to this value, we will adopt this estimated thermal conductivity in this paper.

\section{The effect of the time step on the results}

The numerical approach gives only a discrete approximation of the solution for heat conduction, and for the estimate of the thermal conductivity as well. Consequently, the choice of the time step is of great importance in regard to the quality of the estimated value. Generally, errors of the estimates increase as the time step increases, but using very short time steps is a very time-consuming procedure. According to the results given in Fig. 4 (example for a dry soil sample), time steps of 6 largely reduce the values of the residuals between calculated temperatures and experimental data. Thus, the values of the residuals $\left(0.04^{\circ} \mathrm{C}\right)$ are of the same order of magnitude as the measurement errors given by the temperature probe. According to these results, it seems that the chosen numerical approach with time steps of $6 \mathrm{~s}$ gives a satisfactory solution for estimating the heat conductivity corresponding to the soil. As a matter of fact, this criterion for the choice of the time step (comparison between residuals and measurement errors) is convenient, because even if the modeling is perfect, it is impossible to eliminate in the

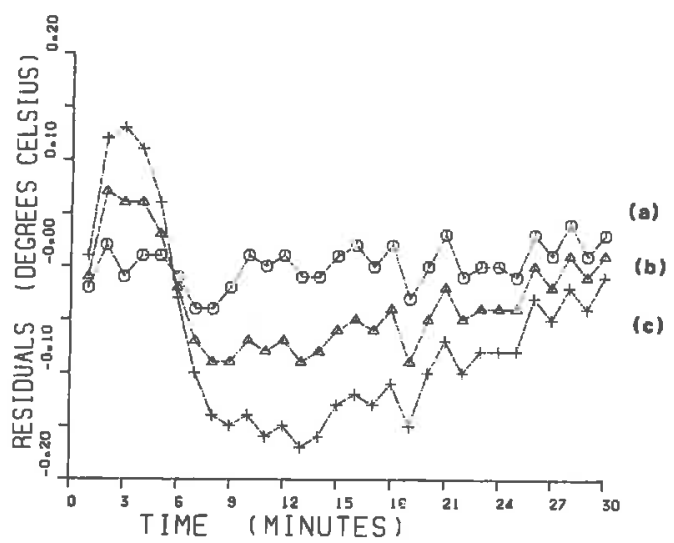

FIG. 4. Effect of the time steps on the residuals: (a) $6 \mathrm{~s}$, (b) $30 \mathrm{~s}$, (c) $60 \mathrm{~s}$.

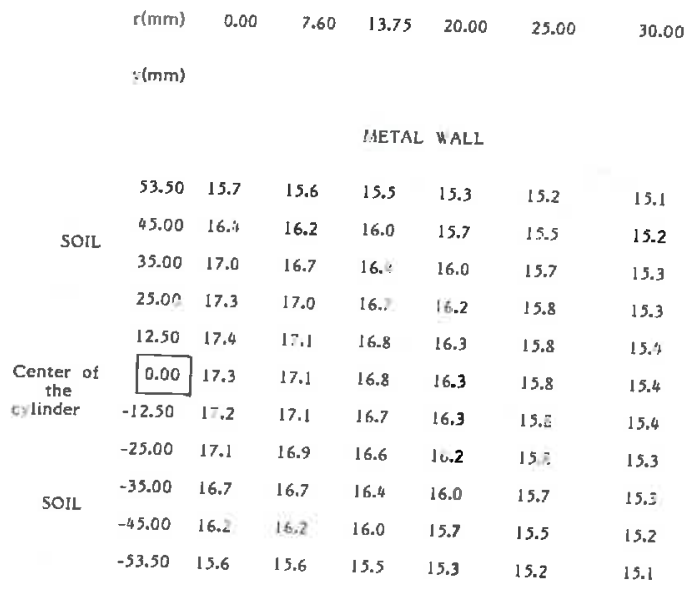

MET.AL W.ALL

Fig. 5. Map of the temperature $\left({ }^{\circ} \mathrm{C}\right)$ in the soil sample after 10 min (initial uniform temperature: $\left.20^{\circ} \mathrm{C} ; \lambda_{r}=0.8 \mathrm{~W} \mathrm{~m}^{-1} \mathrm{~K}^{-1} ; \lambda_{y}=0.7 \mathrm{~W} \mathrm{~m}^{-1} \mathrm{~K}^{-1}\right)$.

residuals the part due to the measurement errors.

\section{Axial and radial heat fluxes}

As previously mentioned, the axial heat flux is generally considered negligible, the assumption being that the metal cylinder is small in diameter and thus the radial flux dominant. In our analysis of this point, the map of soil temperature distribution (Fig. 5) clearly shows that nonnegligible temperature gradients exist in the $O_{r}$ direction of radial heat flux, as well as the $O_{y}$ direction of axial heat flux. It is apparent therefore that this is the general case, granted that different orders of magnitude exist between the two. The numerical technique using finite elements has the advantage of being able to simultaneously take these two heat fluxes without difficulty in regard to the calculation procedure. Using our experimental equipment, note that the assumption of negligible axial heat conduction is unrealistic even in the case where $\lambda_{r}>\lambda_{y}$ (here $\lambda_{r}=0.8 \mathrm{~W} \mathrm{~m}^{-1} \mathrm{~K}^{-1}, \lambda_{y}=0.7 \mathrm{~W} \mathrm{~m}^{-1} \mathrm{~K}^{-1}$ ). Furthermore, observe that the temperature along the ordinate axis is not symmetrically distributed. This is due to the effect of the wire of the probe, which quickly transfers a part of the heat flux toward the base of the metal cylinder.

\section{Sensitivity of the numerical modeling}

It is of great importance to have an idea of the sensitivity of the modeling, that is to say, 
the numerical effect of a given value of thermal conductivity upon the calculated temperatures at the center of the cylinder. Figure 6 compares the calculated values of the temperatures for two different thermal conductivities $\left(0.70 \mathrm{~W} \mathrm{~m}^{-1}\right.$ $\mathrm{K}^{-1}$ and $0.75 \mathrm{~W} \mathrm{~m}^{-1} \mathrm{~K}^{-1}$ ) that differ by approximately $6 \%$. The differences between the two temperatures at a given time have an order of magnitude of $0.25^{\circ} \mathrm{C}$. These differences are greater than the mean value of the residuals already noted $\left(0.04^{\circ} \mathrm{C}\right)$. Consequently, it makes it conveniently possible to separate close values of thermal conductivity in light of the sensitivity of the modeling.

\section{RESULTS}

\section{Estimating the thermal conductivity for an isotropic soil}

Assuming an isotropic porous medium, i.e., $\lambda_{r}$ $=\lambda_{y}$, experiments and calculations have been made to estimate the thermal conductivity for a given soil (clay $=27.2 \%$; silt $=61.7 \%$; sand $=$ $11 \%$; organic matter $=1.7 \%$ ), but having different physical properties. Table 2 presents results from 14 soil samples, with experimental temperatures at the center of the cylinder collected at 1 -min time intervals over a 10 -min period. Samples 1-9 are not compacted, whereas samples

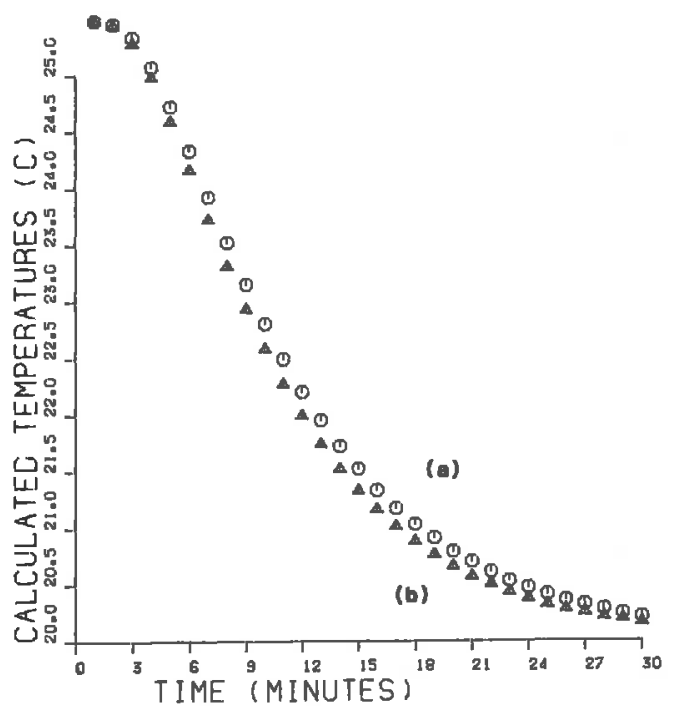

FIG. 6. Effect of different thermal conductivities on the calculated temperatures at the center of the cylinder: (a) $\lambda=0.70 \mathrm{~W} \mathrm{~m}^{-1} \mathrm{~K}^{-1}$, (b) $\lambda=0.75 \mathrm{~W} \mathrm{~m}^{-1}$ $\mathbf{K}^{-1}$.
10-14 are carefully compacted using a piston as previously described.

Two observations emerge after considering the results in Table 2 .

1. In spite of a great variation in the water content and the bulk density, the absolute value of the mean of the temperature residuals is less than or equal to $0.02^{\circ} \mathrm{C}$. Thus, the mean of the temperature residuals is not always equal to 0 , but is very small.

2. Except for the most compacted soil (sample 14), the standard deviation of the residuals is less than or equal to $0.04^{\circ} \mathrm{C}$; that is to say, it has the same order of magnitude as the measurement errors.

The method appears to give reasonable results in terms of calculated thermal conductivities using the numerical approach. For example, the comparison between calculated and experimental temperature profiles in field conditions (bare soil) gives very satisfactory results when calculated values of thermal conductivities are used in heat and mass transfers modeling according to this numerical method. The difference between calculated and experimental temperature in the $25-\mathrm{cm}$ soil layer is less than or equal to $2^{\circ} \mathrm{C}$ for temperatures varying between $15-38^{\circ} \mathrm{C}$ during a week (Bruckler et al. 1986).

Thus, except for the most compacted soil sample, the corresponding estimated values for thermal conductivity are considered satisfactory, and the chosen modeling assumption $\left(\lambda_{r}=\lambda_{y}\right)$ seems adequate under isotropic soil conditions. This assumption also seems to be realistic where the soil samples are either not compacted or are carefully compacted with only slightly reduced bulk densities.

Taking into account the compacted and noncompacted soil samples (1-14), thermal conductivity values have been compared for either a 10-min experiment or for a longer duration, i.e., until the thermal equilibrium in the whole cylinder has been reached in the second water bath (this time varies from 32-72 $\mathrm{min}$ ).

For each sample, Fig. 7 compares the pairs of computed values. Differences between the values of the two thermal conductivities for a given soil sample are less than or equal to $0.02 \mathrm{~W} \mathrm{~m}^{-1}$ $\mathrm{K}^{-1}$. Thus, it is possible to conclude that the effect of the heat transfer due to the vapor phase is probably reduced and the water content distribution in the soil sample stays homogeneous 


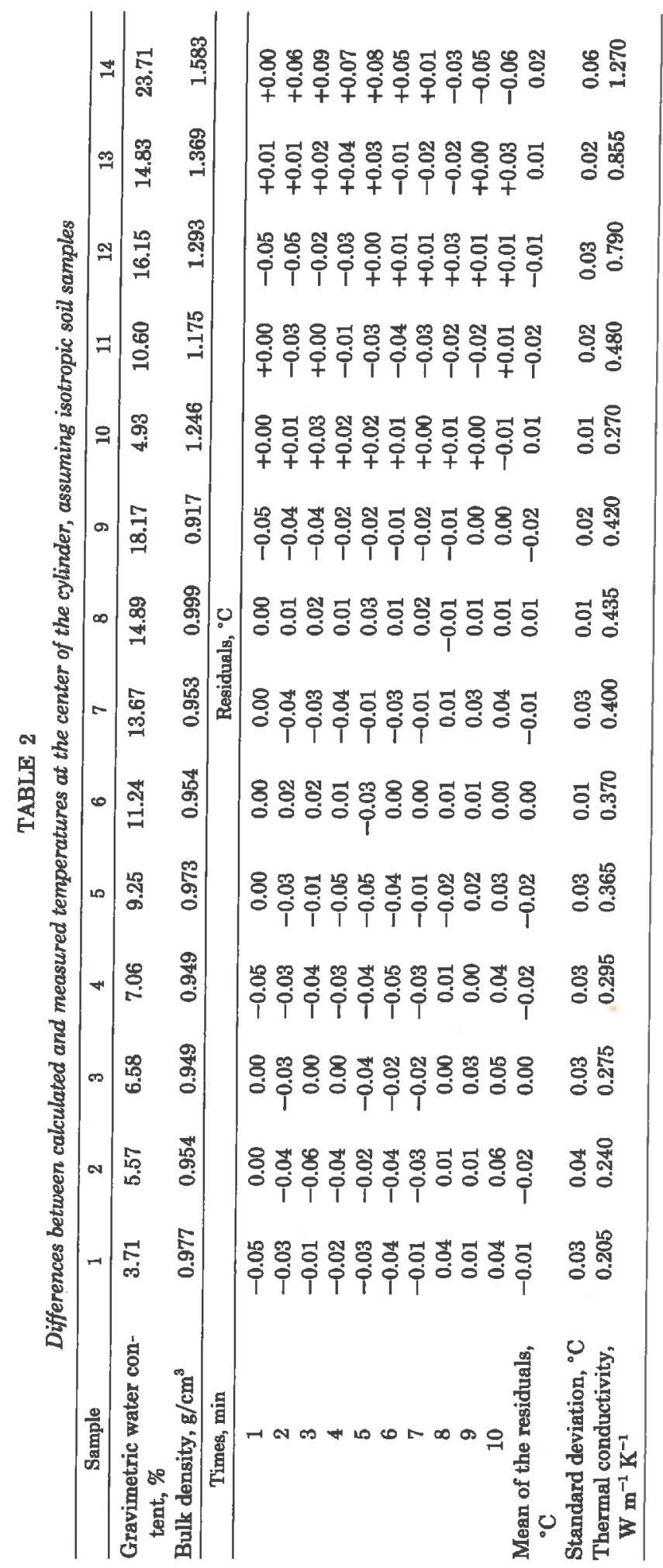




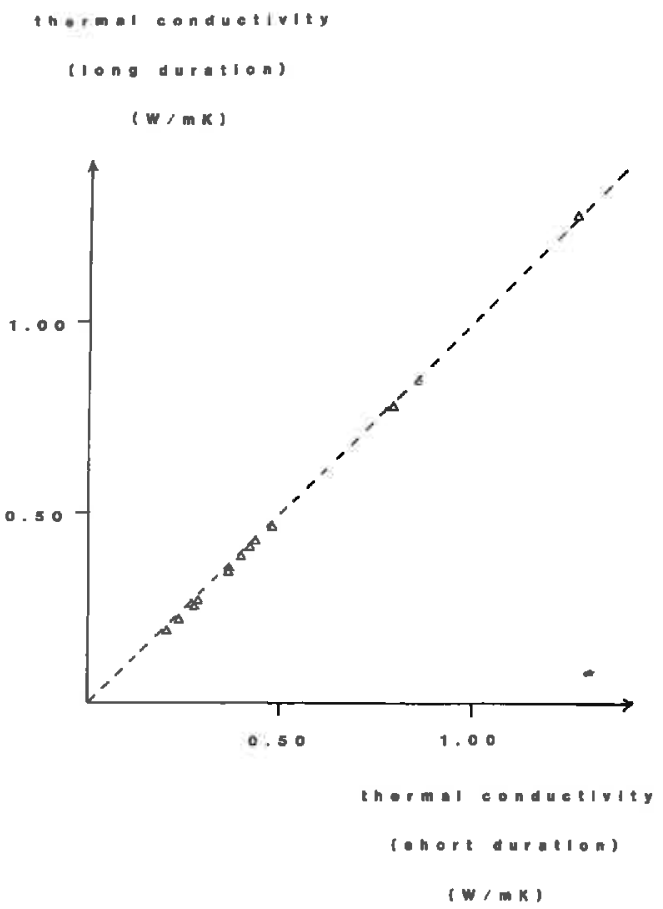

FIG. 7. Relation between the thermal conductivities using short or long experiments. ---, first bisector.

as well, according to the duration of the experiments.

In conclusion, when short experiments and slightly compacted soil samples are used, the values of the temperature residuals verify that the modeling is adequate. For the most compacted soil, being likely the most anisotropic and possessing structural layers, a new mathematical procedure according to anisotropic porous media $\left(\lambda_{r} \neq \lambda_{y}\right)$ has to be taken into account.

\section{Estimating thermal conductivity for an anisotropic soil}

In this section, we present a theoretical and numerical approach without experimental data in the case where $\lambda_{r} \neq \lambda_{y}$. The most important problems to solve are

1. Is it possible to obtain an estimation of the two values of the thermal conductivities $\left(\lambda_{r}, \lambda_{y}\right)$, using only one temperature probe located at the center of the cylinder, or should we use two temperature probes at different locations in the soil sample inside the metal wall?
2. If we need two temperature probes located in the soil, i.e., two data sets of experimental temperatures for a given experiment, where should the probes be located to give a satisfactory estimate of the thermal conductivities?

For these purposes, the numerical approach using the finite element method is combined with a nonlinear fitting method, and the results are presented on the basis of several simulations. The nonlinear fitting method combines the Gauss and the steepest descent methods to find the pair of parameters $\hat{\lambda}_{r}, \hat{\lambda}_{y}$ that minimizes the difference between calculated and experimental values the most (Bard 1974).

\section{Procedure of calculations}

Using the values $\lambda_{r}=0.8 \mathrm{~W} \mathrm{~m}^{-1} \mathrm{~K}^{-1}$ and $\lambda_{y}=$ $0.7 \mathrm{~W} \mathrm{~m}^{-1} \mathrm{~K}^{-1}$, we have generated numerous maps of the temperature distribution in the soil sample (see Fig. 5) with time interval of $1 \mathrm{~min}$. Thus, these calculated values of the temperature at different locations in the soil according to time intervals of $1 \mathrm{~min}$ are regarded as an "experiment" for a soil that has a "real" value of $\lambda_{r}$ $=0.8 \mathrm{~W} \mathrm{~m}^{-1} \mathrm{~K}^{-1}$ and $\lambda_{y}=0.7 \mathrm{~W} \mathrm{~m}^{-1} \mathrm{~K}^{-1}$. For example, for two given locations in the soil sample, calculated values of the temperature are considered as experimental data with two temperature probes located at the same points and for the same time steps. Following this simulation phase, we enter an estimation phase by taking initial values of the parameters $\lambda_{o r}=1$. $\mathrm{W} \mathrm{m} \mathrm{m}^{-1} \mathrm{~K}^{-1}, \lambda_{\text {oy }}=1 \cdot \mathrm{W} \mathrm{m}^{-1} \mathrm{~K}^{-1}$, and using a nonlinear fitting and iterative approach as described before. We then calculate the best estimated values of the parameters $\hat{\lambda}_{r}, \hat{\lambda}_{y}$ that minimize the differences between the calculated and "experimental" temperatures. If we find $\hat{\lambda}_{r} \simeq \lambda_{r}$ and $\hat{\lambda}_{y} \simeq \lambda_{y}$, that makes it possible to conclude that the procedure used for the estimation is acceptable. Note that for all the simulations, the "experimental" values are rounded with a precision of only $0.10^{\circ} \mathrm{C}$. In this manner, the measurement errors $\left(0.05^{\circ} \mathrm{C}\right)$ are taken into account.

\section{Results}

Table 3 compares the estimated values of the parameters $\hat{\lambda}_{r}, \hat{\lambda}_{y}$ for different locations of the temperature probes (Fig. 8).

1. Case 1 corresponds to the same location of the two temperature probes at the center of the cylinder. This case corresponds, in fact, to the 
TABLE 3

Estimated values of the radial thermal conductivity $\left(\lambda_{F}\right)$ and axial thermal conductivity $\left(\lambda_{y}\right)$ for different locations of the temperature probes

\begin{tabular}{lcccccccc}
\hline \multicolumn{1}{c}{ Trial } & 1 & 2 & 3 & 4 & 5 & 6 & 7 & 8 \\
\hline $\begin{array}{l}\text { Locations of the } \\
\text { probes }\end{array}$ & $(\mathrm{a}, \mathrm{a})$ & $(\mathrm{a}, \mathrm{b})$ & $(\mathrm{a}, \mathrm{c})$ & $(\mathrm{a}, \mathrm{d})$ & $(\mathrm{a}, \mathrm{e})$ & $(\mathrm{a}, \mathrm{f})$ & $(\mathrm{a}, \mathrm{g})$ & $(\mathrm{f}, \mathrm{g})$ \\
$\lambda_{r}$ & & & & & & & & \\
{$\left[\lambda_{r}\right]$} & 0.796 & 0.797 & 0.798 & 0.799 & 0.799 & 0.799 & 0.791 & 0.791 \\
& 0.789 & 0.790 & 0.791 & 0.793 & 0.793 & 0.794 & 0.784 & 0.774 \\
$\lambda_{y}$ & 0.803 & 0.805 & 0.805 & 0.805 & 0.804 & 0.805 & 0.797 & 0.808 \\
{$\left[\lambda_{y}\right]$} & 0.785 & 0.738 & 0.727 & 0.708 & 0.704 & 0.712 & 0.818 & 0.715 \\
& 0.694 & 0.641 & 0.669 & 0.676 & 0.684 & 0.692 & 0.726 & 0.693 \\
$r$ & 0.876 & 0.835 & 0.786 & 0.740 & 0.724 & 0.731 & 0.909 & 0.737 \\
& -0.84 & -0.86 & -0.84 & -0.73 & -0.55 & -0.33 & -0.80 & -0.51 \\
\hline
\end{tabular}

situation where we have only one temperature probe.

2. Cases $2,3,4,5$, and 6 correspond to different locations of the two temperature probes. One probe is always at the center of the soil sample, and another is placed along the ordinate axis, as indicated in Fig. 8.

3. Case 7 corresponds to a case where the two probes are along a radius of the cylinder, the first probe being located at the center of the soil sample.

4. Case 8 presents the last situation where the two probes are located near the metal wall along the two axis $\left(O_{r}, O_{y}\right)$.

According to the results of Table 4, it appears that:

1. In case number 1 , the best estimated value for $\hat{\lambda}_{y}$ is 0.78 instead of $0.7 \mathrm{~W} \mathrm{~m}^{-1} \mathrm{~K}^{-1}$. Furthermore, its confidence interval is large, as is the correlation coefficient between the two parameters. Thus, it is difficult to have a satisfactory estimation of the two parameters with only one data set, i.e., one location of the probe at the center of the cylinder. This is a comprehensible result, because heat conduction is more important in the radial direction, and thus the modeling has a greater sensitivity to the $\lambda_{r}$ value than the $\lambda_{y}$ value.

2. In case 7 , the estimated value of $\hat{\lambda}_{y}$ is 0.82 instead of $0.7 \mathrm{~W} \mathrm{~m}^{-1} \mathrm{~K}^{-1}$, and the real value 0.7 exceeds the confidence interval. Thus, the correct estimation is really impossible, and this fact is physically logical because it seems unrealistic to have a good estimation of the heat conductivity corresponding to the axial heat flux if the two probes are only in the radial direction. Perhaps it would be possible to obtain a satisfactory estimation with temperature probes having a high precision, $\left(<0.01^{\circ} \mathrm{C}\right)$ and with a high-precision modeling as well.

3. The best probe locations correspond to case 6 (or 5), having satisfactory estimated values very close to the "real" values, small confidence intervals, and a low coefficient correlation as well. Here the two probes are well separated along the ordinate axis. For cases 2-6, observe how the absolute value of the correlation coefficient decreases as the distance between the two probes increases.

Figure 9 gives the maps of the sum of the squares of the residuals for the contrasting cases 1,6 , and 7 . Therefore, it clearly appears that the effect of the locations of the two temperature probes exerts a great influence on the calculated values of $\lambda_{r}, \lambda_{y}$. The maps corresponding to cases 1 and 7 are very similar; however the map for case 6 is considered closer to the optimum.

Note that the location of the second probe is the unique difference among these three cases, yet the number of temperature values, the measurement errors, the duration of the preliminary simulated "experiment," and the modeling are the same. Thus, the numerical approach employed leads to optimizing the experimental apparatus when the $\lambda_{r}$ and $\lambda_{y}$ values are supposed to be significantly different.

\section{CONCLUSIONS}

In the isotropic case $\left(\lambda_{r}=\lambda_{y}\right)$, it is easy to estimate the thermal conductivity of a soil sample using a numerical approach, except when the porous medium is densely compacted and has structural layers. In the anisotropic case $\left(\lambda_{r} \neq\right.$ $\lambda_{y}$ ), it is possible to estimate two values of the thermal conductivity, having two temperature probes in the soil sample and combining a nu- 


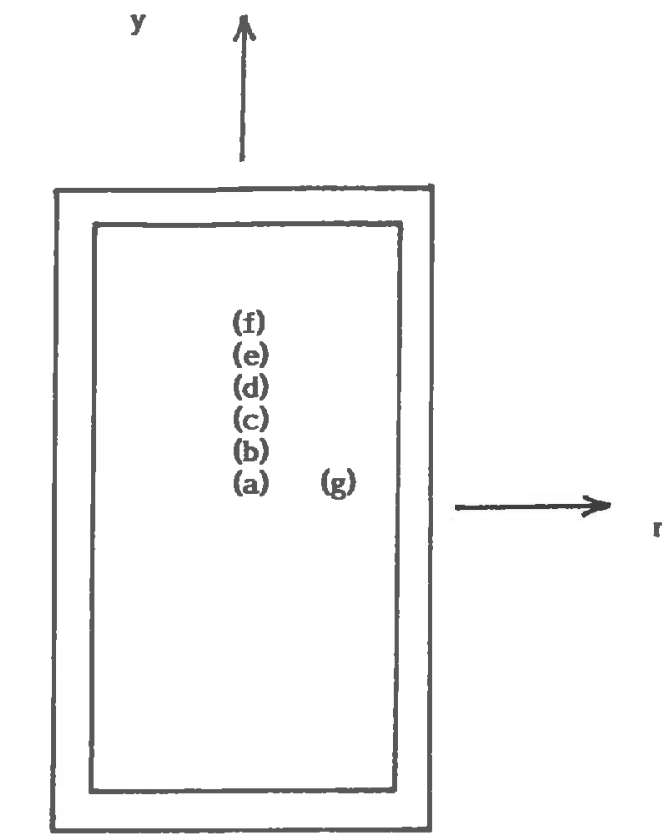

have $\lambda_{i}=f\left(r_{i}, y_{i}\right)$, but only a physically acceptable hypothesis is what is sought.

Finally, improving the measurement methods seems very important if we want to be able to predict heat flow in real field conditions. In the

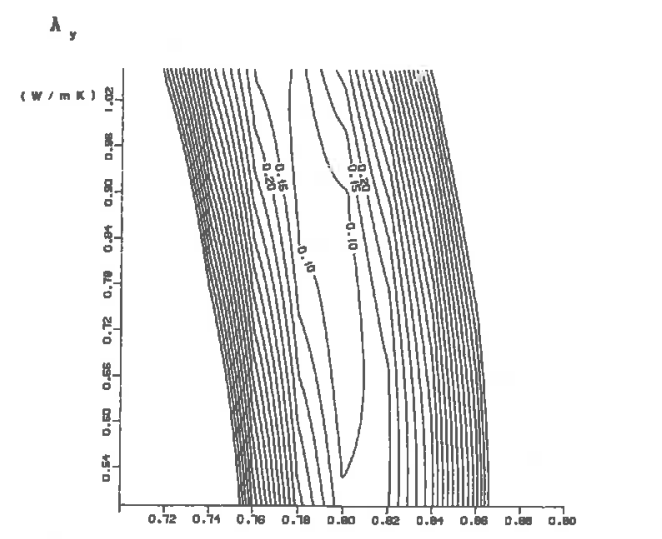

A. (w, m K)

\begin{tabular}{c|c|c} 
Locations & $\begin{array}{l}\mathrm{O}_{\mathrm{r}} \\
(\mathrm{mm})\end{array}$ & $\begin{array}{c}\mathrm{O}_{\mathbf{y}} \\
(\mathrm{mm})\end{array}$ \\
\hline (a) & 0.00 & 0.00 \\
(b) & 0.00 & 12.50 \\
(c) & 0.00 & 25.00 \\
(d) & 0.00 & 35.00 \\
(e) & 0.00 & 45.00 \\
(f) & 0.00 & 53.50 \\
(g) & 13.75 & 0.00
\end{tabular}

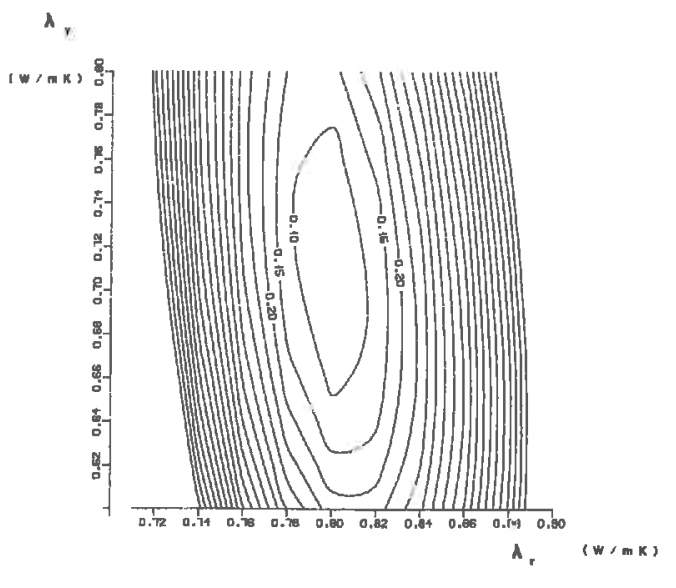

FrG. 8. Different locations of the probes in the cylinder according to the anisotropic case.

merical approach with a nonlinear fitting method; in this case, we suppose always that an anisotropic soil sample may be considered as having only two thermal conductivities $\left(\lambda_{r}, \lambda_{y}\right)$. In fact, in the real case, it is possible to consider that at each point in the porous medium, we

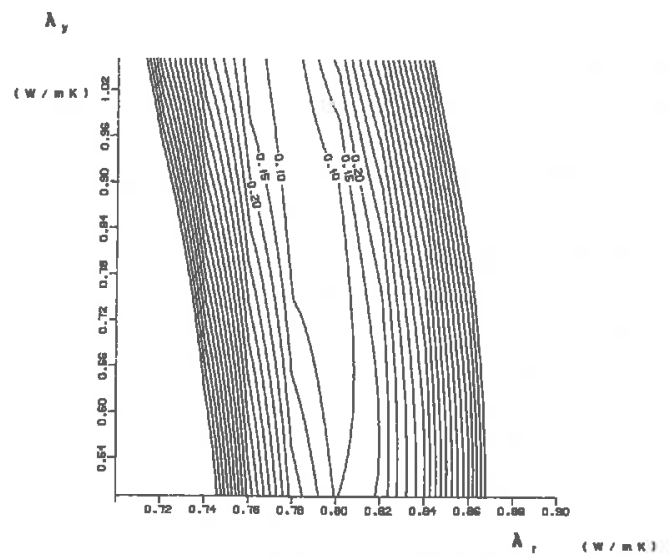

FIG. 9. Maps of the sums of the squares of the temperature residuals for different locations of the probe: top, locations ( $a, a)$; middle, locations ( $a, f$ ); bottom, locations $(a, g)$. 
same way, a comprehensive approach to the variations of thermal conductivity versus water content or bulk density needs at least experimental apparatus having a high precision in both the isotropic and anisotropic cases.

\section{REFERENCES}

Bard, Y. 1974. Nonlinear parameter estimation. Academic, New York.

Barnes, P. L. A. G. Smajstrla, and D. L. Reddell. 1981. Thermal conductivity related to moisture content in soils. Winter Meeting American Society of Agricultural Engineers, paper 81-2511.

Bruckler, L., P. Bertuzzi, J. C. Gaudu, Y. Brunet, A. Passerat de Silans, P. Balabanis, J. L. Thony, and M. Vauclin. 1987. Transferts de masse et de chaleur entre le sol et l'atmosphère: Comparaison entre modélisation et expérience in-situ. In Les Colloques de l'I.N.R.A., 1986 (forthcoming).

Chavent, G. 1971. Analyse fonctionnelle et identification de coefficients répartis dans les équations aux dérivées partielles. Thèse Doctorat es Sciences, Paris.

De Vries, D. A. 1963. Thermal properties of soils. In Physics of plant environment. W. R. Van Wÿk (ed.). North-Holland, Amsterdam, pp. 210-235.

Goldsmid, H. J., E. Kerrie, D. Davies, and V. Papazian. 1981. Probes for measuring the thermal conductivity of granular materials. J. Phys. E: Sci. Instrum. 14:1149-1152.

Moench, A. F., and D. D. Evans. 1970. Thermal conductivity and diffusivity of soil using a cylindrical heat source. Soil Sci. Am. Proc. 34:377-381.

Magar, S. S., S. Kar, and T. K. Subramanyam. 1979. Evaluation of heat flux and thermal characteristics of black soil in relation to soil moisture content during drying. J. Indian Soc. Soil Sci. 27(4):355-360.

Pande, R. N. and D. R. Chaudhary. 1985. Study of thermal conductivity and moisture content of loose materials. Colloquium on energy flux at the soil atmosphere interface. Trieste, Italy.

Parikh, R. J., J. A. Havens, and H. D. Scott. 1979. Thermal diffusivity and conductivity of moist porous media. Soil Sci. Soc. Am. J. 43:1050-1052.

Rama Mohan, R. V., and K. Raghavendra. 1973. Effect of soil compaction on thermal properties of soils of Mysore State. Mysore J. Agric. Sci. 7:238-246.

Sepaskhah, A. R., and L. Boersma. 1979. Thermal conductivity of soils as a function of temperature and water content. Soil Sci. Soc. Am. J. 43:439444. 